\title{
SOIL ORGANIC-MATTER IN WATER-STABLE AGGREGATES UNDER DIFFERENT SOIL-MANAGEMENT PRACTICES
}

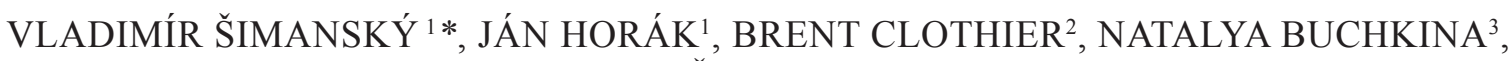 \\ DUŠAN IGAZ ${ }^{1}$
}

${ }^{1}$ Slovak University of Agriculture in Nitra, Slovak Republic

${ }^{2}$ Food Industry Science Centre, New Zealand

${ }^{3}$ Agrophysical Research Institute of the Russian Academy of Agricultural Sciences, Russia

ŠIMANSKÝ, V. - HORÁK, J. - CLOTHIER, B. - BUCHKINA, N. - IGAZ, D.: Soil organic-matter in water-stable aggregates under different soil-management practices. Agriculture (Pol'nohospodárstvo), vol. 63, 2017, no. 4, pp. $151-162$.

An experiment of different management practices in a commercial vineyard, which was established in 2006 in the locality of Nitra-Dražovce, Slovakia on Rendzic Leptosol, was used to evaluate the dynamics of soil organic-matter parameters during the years 2008-2015. The following treatments were established: 1. G (grass without fertilisation as control), 2. T (tillage), 3. T+FYM (tillage + farmyard manure), 4. G+NPK3 (grass $+3^{\text {rd }}$ intensity of fertilisation for vineyards: it means $125 \mathrm{~kg} / \mathrm{ha} \mathrm{N}$, $50 \mathrm{~kg} / \mathrm{ha} \mathrm{P}, 185 \mathrm{~kg} / \mathrm{ha} \mathrm{K}$ ), and 5. G+NPK1 (grass + 1 it intensity of fertilisation for vineyards: it means $100 \mathrm{~kg} / \mathrm{ha} \mathrm{N}, 30 \mathrm{~kg} / \mathrm{ha} \mathrm{P}$, $120 \mathrm{~kg} / \mathrm{ha} \mathrm{K}$ ). The results showed that the soil-management practices in the vineyard significantly influenced the soil organic carbon in water-stable aggregates (SOC in WSA). The content of SOC in WSA increased on average in the following order: T $<\mathrm{G}<\mathrm{G}+\mathrm{NPK} 1<\mathrm{G}+\mathrm{NPK} 3<\mathrm{T}+\mathrm{FYM}$. Intensive soil cultivation in the $\mathrm{T}$ treatment resulted in a statistically significant build-

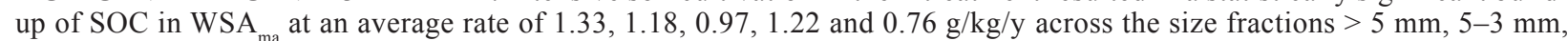
$2-1 \mathrm{~mm}, 1-0.5 \mathrm{~mm}$ and $0.5-0.25 \mathrm{~mm}$, respectively. The content of non-labile carbon reflected the contents of SOC in WSA. The highest labile carbon $\left(\mathrm{C}_{\mathrm{L}}\right)$ in $\mathrm{WSA}_{\text {ma }}$, as compared to others, was found in T+FYM. Overall, application of higher NPK doses resulted in higher content of $\mathrm{C}_{\mathrm{L}}$ in $\mathrm{WSA}_{\mathrm{ma}}$ compared with the lower applications of NPK. On the other hand, lower applications of NPK to soil increased the content of $\mathrm{C}_{\mathrm{L}}$ in $\mathrm{WSA}_{\text {mi }}$, as compared to G+NPK3.

Key words: soil organic carbon, labile carbon, non-labile carbon, soil structure, soil fertility

Soil organic-matter (SOM) plays an important role in plant-nutrient cycling, increasing yields and improving the physical, chemical and biological properties of soils (Bhattacharyya et al. 2010; Gaida et al. 2013) and the impact varies across space and time and is a result of the influence of many environmental and anthropogenic factors (Jonczak 2014).
For example, intensive cultivation leads to loss of SOM (Khorramdel et al. 2013) while reduced tillage increases SOM (Šimanský et al. 2008). The results of Tong et al. (2014) indicated that application of manure had the highest sequestration rate of total SOC in comparison to mineral fertilisers. Triberti et al. (2008) found that continuous additions of organ-

doc. Ing. Vladimír Šimanský, PhD. (*Corresponding author), Department of Soil Science, FAFR - SUA Nitra, 94976 Nitra, Tr. A. Hlinku 2, Slovak Republic. E-mail: Vladimir.Simansky@uniag.sk

Ing. Ján Horák, PhD., Department of Biometeorology and Hydrology, HLEF - SUA Nitra, 94976 Nitra, Hospodárska 7 , Slovak Republic. E-mail: jan.horak@uniag.sk

Dr. Brent E. Clothier, Plant and Food Research, Food Industry Science Centre, Bachelor Road, PO Box 11-600, Palmerston North 4442, New Zealand. E-mail: Brent.Clothier@plantandfood.co.nz

Dr. Natalya P. Buchkina, Agrophysical Research Institute of the Russian Academy of Agricultural Sciences, 14 Grazhdansky prospect, St. Petersburg, Russia. E-mail: buchkina_natalya@mail.ru

doc. Ing. Dušan Igaz, PhD., Department of Biometeorology and Hydrology, HLEF - SUA Nitra, 94976 Nitra, Hospodárska 7, Slovak Republic. E-mail: dusan.igaz@uniag.sk 
ic material to the soil led to a SOC build up at rates $0.16-0.26 \mathrm{t} \mathrm{C} / \mathrm{ha} / \mathrm{y}$ over a 22 year period. Purakayastha et al. (2008) reported the rates of SOC build-up in the soil up to a level of $1.0 \mathrm{Mg} / \mathrm{ha} / \mathrm{y}$ due to NPK + farmyard application under a maize-wheat-cowpea cropping system. Abdollahi et al. (2014) showed the rate of SOC due to added organic fertilisers to be between $220-240 \mathrm{~kg} \mathrm{C} / \mathrm{ha} / \mathrm{y}$. All the information about the increase or decline of $\mathrm{C}$ is from different soils. However, the determination of carbon pools in aggregates provides important information on soil C sequestration processes and mineralization mechanisms in aggregate size fractions that could be used to protect SOC by using appropriate soil and crop management practices (Whalen \& Chang 2002). Water-stable aggregates may protect the carbon inside of aggregates more intensively than aggregates with low resistance against destructive influence of water (Šimanský \& Bajčan 2014) which is also an effective strategy to mitigate global climate change (Paustian et al. 1997), whilst sustaining soil health. Changes in land use significantly influence the carbon cycle. An important factor of SOM stabilization is a favourable soil structure (Berhe \& Kleber 2013; Chaplot \& Cooper 2015). This is because SOM is one of the most significant binding agents which is responsible for the association of mineral particles within the aggregates (Rabbi et al. 2015).

Vineyard soils are strongly influenced by anthropogenic activities. Before establishing a vineyard, the original soil type is transformed. There is a change in the structural conditions and capacity of the soil to retain the organic carbon. Therefore, understanding the dynamics and mechanism of carbon sequestration in the water-stable aggregates under different management practices in vineyard soils could be a primary way to improve the soil fertility. This will sustain the grape yield production around the world, and in Slovakia in particular.

The objectives of our study were: (i) to determine $\mathrm{C}$ changes in the size fractions of water-stable aggregates under the different management practices in a vineyard; (ii) to determine the rate of $\mathrm{C}$ input into water-stable aggregates under different management practices over the period of 8 years.

\section{MATERIAL AND METHODS}

The study was carried out in a commercial vineyard located in Nitra-Dražovce $\left(48^{\circ} 21^{\prime} 6.16^{\prime \prime} \mathrm{N}\right.$; $\left.18^{\circ} 3^{\prime} 37.33^{\prime \prime} \mathrm{E}\right)$ in Nitra wine-growing region of Slovakia. The climate is temperate with an average annual rainfall of $550 \mathrm{~mm}$ and with the mean annual temperature being $\geq 10^{\circ} \mathrm{C}$. The soil had developed on limestone and dolomite and is classified as Rendzic Leptosol (WRB 2014). The soil was analysed prior the vineyard establishment (spring 2000 ) and contained $17.0 \pm 1.6 \mathrm{~g} / \mathrm{kg}$ of soil organic carbon, $1867 \pm 103 \mathrm{mg} / \mathrm{kg}$ of total nitrogen and had a $\mathrm{pH}_{\mathrm{H} 2 \mathrm{O}}$ value of $7.18 \pm 0.08$, with the base saturation percentage being $99.3 \pm 0.01 \%$. The content of

T a b

The various treatments imposed in the vineyard (Nitra-Drážovce)

\begin{tabular}{|c|c|}
\hline Treatment & Description \\
\hline Control (G) & Sown grass in the rows and between vine rows, without fertilisation. \\
\hline Tillage $(\mathrm{T})$ & $\begin{array}{l}\text { Every year medium tilth to the depth of } 25 \mathrm{~cm} \text { with intensive cultivation (three times on } \\
\text { average, without fertilisation) between vine rows during the growing season. }\end{array}$ \\
\hline $\begin{array}{l}\text { Tillage }+ \text { application of farmyard } \\
\text { manure }(\mathrm{T}+\mathrm{FYM})\end{array}$ & $\begin{array}{l}\text { Medium tilth to the depth of } 25 \mathrm{~cm} \text { with application of farmyard manure (FYM) in a dose of } \\
40 \text { t/ha applied in autumn } 2005,2009 \text { and } 2012 \text { with intensive cultivation between vine rows } \\
\text { during growing season. }\end{array}$ \\
\hline $\begin{array}{l}\text { Application of fertilisers in } 3^{\text {rd }} \\
\text { intensity for vineyards according } \\
\text { to Fecenko and Ložek (2000) } \\
\text { (G+NPK3) }\end{array}$ & $\begin{array}{l}\text { There was used Duslofert Extra 14-10-20-7 fertiliser with the real doses of nutrients applied in } \\
\text { the treatment being } 125 \mathrm{~kg} / \mathrm{ha} \mathrm{N}, 50 \mathrm{~kg} / \mathrm{ha} \mathrm{P}, 185 \mathrm{~kg} / \mathrm{ha} \mathrm{K} \text {. Split application of fertilisers was } \\
\text { applied with } 2 / 3 \text { of fertilisers applied in the spring (bud burst - on March) and } 1 / 3 \text { during the } \\
\text { flowering stage (on May). The grass was sown in and between the vine rows. }\end{array}$ \\
\hline $\begin{array}{l}\text { Application of fertilisers in } 1^{\text {st }} \\
\text { intensity for vineyards according } \\
\text { to Fecenko and Ložek (2000) } \\
\text { (G+NPK1) }\end{array}$ & $\begin{array}{l}\text { There was used Duslofert Extra 14-10-20-7 fertiliser with the real doses of nutrients applied } \\
\text { in the treatment being } 100 \mathrm{~kg} / \mathrm{ha} \mathrm{N}, 30 \mathrm{~kg} / \mathrm{ha} \mathrm{P}, 120 \mathrm{~kg} / \mathrm{ha} \mathrm{K} \text {. The doses of nutrients were split } \\
\text { with } 1 / 2 \text { applied in the spring (bud burst - on March) and } 1 / 2 \text { during flowering (on May). The } \\
\text { grass was sown in and between the vine rows. }\end{array}$ \\
\hline
\end{tabular}


sand, silt and clay was $57 \%, 33 \%$ and $10 \%$, respectively.

A variety of grasses (Lolium perenne $50 \%+$ Poa pratensis $20 \%+$ Festuca rubra commutata $25 \%+$ Trifolium repens 5\%) were sown as grass strip between the vineyard rows in 2003. The experiment with different soil management practices (5 treatments) was initiated in 2006, and laid out on a randomized complete block design with four replicates. The investigated treatments are presented in Table 1. Soils samples $(0-0.25 \mathrm{~m})$ were taken from 4 random locations within each treatment every spring during years 2008-2015. Soil samples were then mixed together to form an average sample for each treatment. Large clods were gently broken up along natural fracture lines, and then air-dried in the laboratory to achieve undisturbed soil samples for the determination of the individual size fractions of aggregates. The Baksheev method for aggregate separation was adopted from Vadjunina and Korchagina (1986). Seven aggregate-size fractions were separated by wet-sieving of the soil through the series of six sieves. Briefly, the soil sample ( $30 \mathrm{~g})$ was covered with distilled water with the water level $1 \mathrm{~cm}$ above aggregates. Two hours later, the sample was transferred to the top sieve $(>5 \mathrm{~mm})$ in a cylindrical container (Baksheev device), which had been filled with distilled water. The cylinder was hermetically closed and the sample was sieved for 12 minutes. The size fractions of WSA were the following: $>5$, $5-3,3-2,2-1,1-0.5,0.5-0.25 \mathrm{~mm}$ as water-stable macro-aggregates ( $\mathrm{WSA}_{\mathrm{ma}}$ ) and $<0.25 \mathrm{~mm}$ as micro-aggregates $\left(\mathrm{WSA}_{\mathrm{mi}}\right.$ ). The material retained was quantified in each sieve, except for the micro-aggregates $(<0.25 \mathrm{~mm})$. Their content was calculated as difference between total weight of soil sample and sums of the macro-aggregates $(>0.25 \mathrm{~mm})$. The content of soil organic carbon (SOC) in the individual size fractions of water-stable aggregates (WSA) was determined using the wet combustion method by oxidation of organic matter using a mixture of $0.07 \mathrm{M} \mathrm{H}_{2} \mathrm{SO}_{4}$ and $\mathrm{K}_{2} \mathrm{Cr}_{2} \mathrm{O}_{7}$ with titration using 0.01 $\mathrm{M}$ Mohr's salt. This is described in Dziadowiec and Gonet (1999). The labile carbon content $\left(C_{L}\right)$ was extracted from samples containing $1 \mathrm{~g}$ of individual size fractions of WSA by shaking in $50 \mathrm{~mL}$ of 0.005 $\mathrm{M} \mathrm{KMnO}_{4}$ for $2 \mathrm{~h}$. After centrifugation, the $\mathrm{C}_{\mathrm{L}}$ was determined with titration using $0.05 \mathrm{M}$ Mohr's salt
(Loginow et al. 1987). Non-labile carbon $\left(\mathrm{C}_{\mathrm{NL}}\right)$ was calculated according to equation (1):

$$
C_{N L}=S O C-C_{L}(1)
$$

where: $\mathrm{SOC}$ (in $\mathrm{g} / \mathrm{kg}$ ) is the organic carbon content and $\mathrm{C}_{\mathrm{L}}$ (in $\mathrm{g} / \mathrm{kg}$ ) is the labile carbon content

The statistical processing of the data included checking of the data for normality and later by using one-way analysis of variance (ANOVA) using Statgraphics Centurion XV.I statistical software (Statpoint Technologies, Inc., USA). Significant differences between the treatment means of the three replicates were identified using a least significant difference $(L S D)$ at $p<0.05$. The correlations between SOM in WSA and WSA contents were then determined. A linear model was used to evaluate the trends of SOC, $\mathrm{C}_{\mathrm{NL}}$ and $\mathrm{C}_{\mathrm{L}}$ under the different soil-management practices of vineyard during the period of 8 years.

\section{RESULTS AND DISCUSSION}

\section{Water-stable aggregates}

The distribution of aggregate sizes under the different soil-management practices of the vineyard is shown in Figure 1. If the results of WSA were evaluated as an average of WSA mi $_{\text {and }}$ WSA $_{\text {ma }}$ there would not be any significant differences between management practices. However, if they were evaluated by individual aggregate sizes of $\mathrm{WSA}_{\text {ma }}$ the one-way ANOVA analysis showed significant differences between treatments, for size fractions of WS$\mathrm{A}_{\mathrm{ma}}>5 \mathrm{~mm}, 5-3 \mathrm{~mm}, 1-0.5 \mathrm{~mm}$ and $0.5-0.25 \mathrm{~mm}$. The lowest average content of WSA ma $_{\text {was }}$ determined in $\mathrm{T}$ treatment. There have been reported a negative impacts of intensive soil cultivation on the content of water-stable aggregates (Wang et al. 2015). Tillage was held responsible for the disruption of soil aggregates (Plante \& McGill 2002). On the other hand, the highest average content of WS$A_{\text {ma }}$ was found in the G+NPK3 treatment and then with $\mathrm{G}>\mathrm{T}+\mathrm{FYM}>\mathrm{G}+\mathrm{NPK} 1$. The complexity of the chemical and physical effects of fertilisers has resulted in variable effects of fertilisation on the aggregates. There have been reported negative impacts of the use mineral fertilisers on aggregate resistance (Czachor et al. 2015). However, the fertiliser ap- 
plication generally improves the soil aggregation (Haynes \& Naidu 1998) due to increasing macro-aggregation and enhanced resistance to slaking (Whalen \& Chang 2002).

\section{Soil organic carbon in water-stable aggregates}

Our study has shown that soil-management practices significantly influenced SOC in WSA (Table 2). The content of SOC was lower in WSA $\mathrm{mi}_{\mathrm{m}}$ than in $\mathrm{WSA}_{\mathrm{ma}}$ across all treatments. Six et al. (2004) pointed out that at the lowest depletion of $\mathrm{C}$ content in small macro-aggregates and micro-aggregates, could be due to a better protection of $\mathrm{C}$ in these aggregate sizes (Rabbi et al. 2015). The lowest content of SOC in $\mathrm{WSA}_{\mathrm{mi}}$ was found here in $\mathrm{T}$ and the highest in $\mathrm{G}+\mathrm{NPK} 1$ treatment. There was observed an increase of the $\mathrm{C}$ concentration for larger size fractions of $\mathrm{WSA}_{\mathrm{ma}}$, which is in line with other studies where the linear increase of $\mathrm{C}$ concentration was found to be in larger aggregates size classes (Biswas et al. 2009). Overall, the highest average SOC content in $\mathrm{WSA}_{\mathrm{ma}}$ was in T+FYM, while the lowest ones were in the $\mathrm{T}$ treatment. The SOC content in $\mathrm{WSA}_{\mathrm{ma}}$ increased in the following order: $\mathrm{T}<\mathrm{G}<\mathrm{G}+\mathrm{NPK} 1<\mathrm{G}+\mathrm{NPK} 3$ $<\mathrm{T}+\mathrm{FYM}$. Several authors have also confirmed a loss of C in the soil (Khorramdel et al. 2013), and in the aggregates (Abdollahi et al. 2014), under intensive tillage systems. This is logical because the tillage disrupts the soil and supports mineralization processes in the soils (Polidori et al. 2008). Application of organic and mineral fertilisers increased the average content of SOC in $\mathrm{WSA}_{\mathrm{ma}}$ by $22 \%, 9 \%$ and $6 \%$ in $\mathrm{T}+\mathrm{FYM}, \mathrm{G}+\mathrm{NPK} 3$ and $\mathrm{G}+\mathrm{NPK} 1$, respectively, as compared to control (Table 2).

Table 3 provides the dynamics of SOC at the individual aggregate sizes of $\mathrm{WSA}_{\mathrm{ma}}$ under the different soil-management practices during the whole studied period. Intensive cultivation of the soil in the $\mathrm{T}$ treatment significantly increased the SOC in $\mathrm{WSA}_{\mathrm{ma}}$ at an average rate of $1.33,1.18,0.97,1.22$ and $0.78 \mathrm{~g} / \mathrm{kg} / \mathrm{y}$ across the size fractions $>5 \mathrm{~mm}$, 5-3 mm, 2-1 mm, 1-0.5 $\mathrm{mm}$ and $0.5-0.25 \mathrm{~mm}$, respectively. Expressed as percentage this is an in-

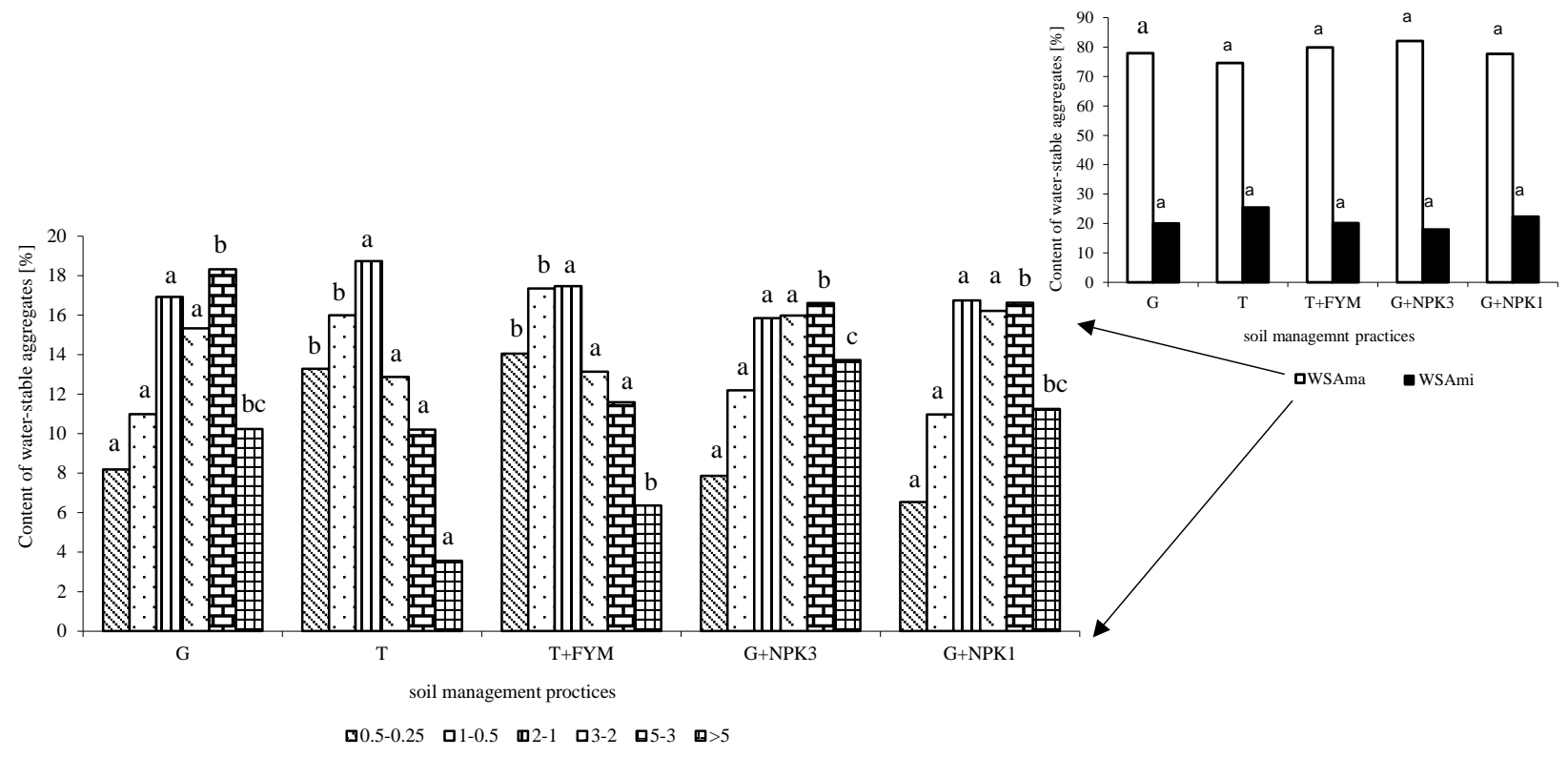

Figure 1. Statistical evaluation of water-stable aggregates contents under different soil-management practices

where: $\mathrm{G}$ - control; T - tillage; T+FYM - tillage+farmyard manure; G+NPK3 - doses of NPK fertilisers in $3^{\text {-rd }}$ intensity for vineyards; G+NPK1 - doses of NPK fertilisers in $1^{\text {st }}$ intensity for vineyards; $\mathrm{WSA}_{\mathrm{ma}}$ - water-stable macro-aggregates; WSA - water-stable micro-aggergates.

Different letters between columns $(\mathrm{a}, \mathrm{b}, \mathrm{c})$ indicate that treatment means are significantly different at $P<0.05$ according to $L S D$ multiple-range test. 
crease of $90,82,81,148$ and $130 \%$ of SOC in these size fractions of $\mathrm{WSA}_{\mathrm{ma}}$ over the period of 8 years. As mentioned above, $\mathrm{C}$ in the soil is reduced due to cultivation. However, its content in the size fractions of $\mathrm{WSA}_{\mathrm{ma}}$ was increased, which means that part of $\mathrm{C}$ after the soil disturbances is sequestered inside of the $\mathrm{WSA}_{\mathrm{ma}}$, especially in the two smallest size fractions ( $1-0.5$ and $0.5-0.25 \mathrm{~mm})$. There was not found to be any significant effect on the build-up of SOC in the individual aggregate sizes of WSA in the case of the application of NPK in the $1^{\text {st }}$ and $3^{\text {rd }}$ intensities of vineyard fertilisation (Table 3). Fertilisers can influence SOM in a different ways. Their use improves residue quantity and quality. But this does not necessarily increase the SOC pool. Tong et al. (2014) reported that the soils under NPK and NP treatments significantly increased SOC stocks. However, fertilisers may also decrease $\mathrm{C}$ content

T $\quad$ a $\quad$ b 1 l e 2

Statistical evaluation of organic and labile carbon contents in size fractions of water-stable aggregates

\begin{tabular}{|c|c|c|c|c|c|c|c|}
\hline \multirow{2}{*}{ Parameters } & \multirow{2}{*}{\multicolumn{2}{|c|}{$\begin{array}{c}\text { Size fractions of water-stable } \\
\text { aggregates in } \mathrm{mm}\end{array}$}} & \multicolumn{5}{|c|}{ Treatments } \\
\hline & & & $\mathrm{G}$ & $\mathrm{T}$ & $\mathrm{T}+\mathrm{FYM}$ & $\mathrm{G}+\mathrm{NPK} 3$ & $\mathrm{G}+\mathrm{NPK} 1$ \\
\hline \multirow{9}{*}{$\begin{array}{l}\underset{\infty 0}{0} \\
\underset{\infty}{\infty} \\
\cup \\
0 \\
\infty\end{array}$} & $\mathrm{WSA}_{\mathrm{mi}}$ & $<0.25$ & $12.2^{\mathrm{b}}$ & $10.0^{\mathrm{a}}$ & $12.1^{\mathrm{b}}$ & $12.6^{\mathrm{b}}$ & $12.7^{\mathrm{b}}$ \\
\hline & & & & & & & \\
\hline & \multirow{6}{*}{$\mathrm{WSA}_{\mathrm{ma}}$} & $0.25-0.5$ & $14.0^{\mathrm{ab}}$ & $12.4^{\mathrm{a}}$ & $16.0^{\mathrm{b}}$ & $15.0^{\mathrm{ab}}$ & $15.0^{\mathrm{ab}}$ \\
\hline & & $0.5-1$ & $16.8^{\mathrm{ab}}$ & $14.5^{\mathrm{a}}$ & $18.8^{\mathrm{b}}$ & $16.3^{\mathrm{ab}}$ & $17.2^{\mathrm{ab}}$ \\
\hline & & $1-2$ & $16.4^{\mathrm{a}}$ & $15.5^{\mathrm{a}}$ & $19.3^{b}$ & $17.2^{\mathrm{ab}}$ & $17.4^{\mathrm{ab}}$ \\
\hline & & $2-3$ & $14.9^{\mathrm{ab}}$ & $14.8^{\mathrm{a}}$ & $17.8^{\mathrm{c}}$ & $17.4^{\mathrm{bc}}$ & $16.3^{\mathrm{abc}}$ \\
\hline & & $3-5$ & $14.9^{\mathrm{a}}$ & $15.9^{\mathrm{ab}}$ & $19.6^{\mathrm{c}}$ & $17.7^{\mathrm{bc}}$ & $16.7^{\mathrm{ab}}$ \\
\hline & & $>5$ & $16.9^{\mathrm{a}}$ & $17.5^{\mathrm{a}}$ & $23.3^{\mathrm{b}}$ & $18.9^{\mathrm{a}}$ & $17.1^{\mathrm{a}}$ \\
\hline & Mean WSA ${ }_{\text {ma }}$ & & $15.7^{\mathrm{a}}$ & $15.1^{\mathrm{a}}$ & $19.1^{\mathrm{c}}$ & $17.1^{\mathrm{b}}$ & $16.6^{\mathrm{b}}$ \\
\hline \multirow{9}{*}{$\begin{array}{l}\overrightarrow{000} \\
\frac{2}{0.00} \\
\overrightarrow{0} \\
\vec{z}\end{array}$} & $\mathrm{WSA}_{\mathrm{mi}}$ & $<0.25$ & $10.6^{\mathrm{b}}$ & $8.37^{\mathrm{a}}$ & $10.3^{\mathrm{b}}$ & $10.6^{\mathrm{b}}$ & $11.0^{\mathrm{b}}$ \\
\hline & \multirow{7}{*}{$\mathrm{WSA}_{\mathrm{ma}}$} & & & & $138^{b}$ & $12 \mathrm{gab}$ & 120 ab \\
\hline & & $\begin{array}{c}0.25-0.5 \\
05-1\end{array}$ & $12.2^{\mathrm{ab}}$ & $10.5^{\circ}$ & $\begin{array}{l}13.8^{\circ} \\
16.3 b\end{array}$ & $\begin{array}{l}12.9^{\mathrm{a}} \\
13 \mathrm{~g}^{\mathrm{ab}}\end{array}$ & $\begin{array}{l}13.0^{\mathrm{a}} \\
148^{\mathrm{ab}}\end{array}$ \\
\hline & & $1-2$ & $145^{\mathrm{ab}}$ & $130^{\mathrm{a}}$ & $166^{\mathrm{b}}$ & $150^{\mathrm{ab}}$ & $15.0 \mathrm{~b}$ \\
\hline & & $1-2$ & 17.0 & 15.0 & 10.0 & 15.0 & 15.4 \\
\hline & & $2-3$ & $13.2^{\mathrm{ab}}$ & $12.8^{\mathrm{a}}$ & $15.5^{\mathrm{c}}$ & $15.1^{b c}$ & $14.2^{\mathrm{abc}}$ \\
\hline & & $3-5$ & $13.0^{\mathrm{a}}$ & 13.8 & $17.1^{\mathrm{b}}$ & $15.1^{\mathrm{ab}}$ & $14.7^{\mathrm{a}}$ \\
\hline & & $>5$ & $14.9^{\mathrm{a}}$ & $15.0^{\mathrm{a}}$ & $20.1^{\mathrm{b}}$ & $16.2^{\mathrm{a}}$ & $15.1^{\mathrm{a}}$ \\
\hline & Mean WSA $_{\text {ma }}$ & & $13.8^{\mathrm{a}}$ & $13.0^{\mathrm{a}}$ & $16.6^{\mathrm{c}}$ & $14.7^{\mathrm{b}}$ & $14.5^{\mathrm{ab}}$ \\
\hline \multirow{9}{*}{$\begin{array}{l}\underset{00}{00} \\
\underset{0}{00} \\
ن \\
ن\end{array}$} & $\mathrm{WSA}_{\mathrm{mi}}$ & $<0.25$ & $1.57^{\mathrm{a}}$ & $1.67^{\mathrm{ab}}$ & $1.80^{\mathrm{ab}}$ & $2.00^{\mathrm{b}}$ & $1.68^{\mathrm{ab}}$ \\
\hline & \multirow{7}{*}{$\mathrm{WSA}_{\mathrm{ma}}$} & & & & & & \\
\hline & & $0.25-0.5$ & $1.76^{\mathrm{a}}$ & $1.93^{\mathrm{ab}}$ & $2.42^{\mathrm{b}}$ & $2.09^{\mathrm{ab}}$ & $2.01^{\mathrm{ab}}$ \\
\hline & & $0.5-1$ & $2.08^{a}$ & $1.93^{\mathrm{a}}$ & $2.44^{\mathrm{a}}$ & $2.42^{\mathrm{a}}$ & $2.43^{\mathrm{a}}$ \\
\hline & & $1-2$ & $1.91^{\mathrm{a}}$ & $2.43^{\mathrm{bc}}$ & $2.66^{\mathrm{c}}$ & $2.20^{\mathrm{ab}}$ & $1.99^{\mathrm{a}}$ \\
\hline & & $2-3$ & $1.73^{\mathrm{a}}$ & $2.11^{\mathrm{ab}}$ & $2.32^{\mathrm{b}}$ & $2.23^{\mathrm{b}}$ & $2.03^{\mathrm{ab}}$ \\
\hline & & $3-5$ & $1.81^{\mathrm{a}}$ & $2.17^{\mathrm{ab}}$ & $2.56^{\mathrm{b}}$ & $2.54^{\mathrm{b}}$ & $1.97^{\mathrm{a}}$ \\
\hline & & $>5$ & $2.08^{a}$ & $1.93^{\mathrm{a}}$ & $2.44^{\mathrm{a}}$ & $2.42^{\mathrm{a}}$ & $2.43^{\mathrm{a}}$ \\
\hline & Mean $\mathrm{WSA}_{\mathrm{ma}}$ & & $1.90^{\mathrm{a}}$ & $2.08^{\mathrm{ab}}$ & $2.47^{\mathrm{b}}$ & $2.32^{\mathrm{b}}$ & $2.14^{\mathrm{ab}}$ \\
\hline
\end{tabular}

where: $\mathrm{SOC}$ - organic carbon in water-stable aggregates; $\mathrm{C}_{\mathrm{NL}}-$ non-labile carbon in water-stable aggregates; $\mathrm{C}_{\mathrm{L}}-$ labile carbon in water-stable aggregates; $\mathrm{WSA}_{\mathrm{mi}}$ - water-stable macro-aggregates; $\mathrm{WSA}_{\mathrm{mi}}$ - water-stable micro-aggregates; $\mathrm{G}$ control; T - tillage; T+FYM - tillage+farmyard manure; G+NPK3 - doses of NPK fertilisers in $3^{\text {rd }}$ intensity for vineyards; G+NPK 1 - doses of NPK fertilisers in $1^{\text {st }}$ intensity for vineyards. Different letters between columns $(a, b$, c) indicate that treatment means are significantly different at $P<0.05$ according to $L S D$ multiple-range test. 
$\begin{array}{lllllllllllll}\mathrm{T} & \mathrm{a} & \mathrm{b} & 1 & \mathrm{e} & 3\end{array}$

Trends of the SOC distribution in the WSA during the 2008-2015 ( $\mathrm{y}=$ SOC content) with time ( $\mathrm{x}=$ years)

\begin{tabular}{|c|c|c|c|}
\hline Treatments & Equations & Probability & Trend \\
\hline \multicolumn{4}{|c|}{$\mathrm{SOC}$ in $\mathrm{WSA}_{\mathrm{ma}}>5 \mathrm{~mm}$} \\
\hline G & $y=0.0071 x+2.5071$ & n.s. & increase \\
\hline $\mathrm{T}$ & $y=1.3333 x-2664.5$ & ++ & increase \\
\hline $\mathrm{T}+\mathrm{FYM}$ & $y=1.2929 x-2577.3$ & n.s. & increase \\
\hline $\mathrm{G}+\mathrm{NPK} 3$ & $y=0.9298 x-1851.3$ & n.s. & increase \\
\hline $\mathrm{G}+\mathrm{NPK} 1$ & $y=0.506 x-1000.6$ & n.s. & increase \\
\hline \multicolumn{4}{|c|}{$\mathrm{SOC}$ in $\mathrm{WSA}_{\mathrm{ma}} 5-3 \mathrm{~mm}$} \\
\hline G & $y=-0.1143 x+244.74$ & n.s. & decrease \\
\hline $\mathrm{T}$ & $y=1.175 x-2347.6$ & + & increase \\
\hline $\mathrm{T}+\mathrm{FYM}$ & $y=0.9774 x-1946.4$ & + & increase \\
\hline $\mathrm{G}+\mathrm{NPK} 3$ & $y=0.2643 x-513.94$ & n.s. & increase \\
\hline $\mathrm{G}+\mathrm{NPK} 1$ & $y=-0.5917 x+1206.8$ & n.s. & decrease \\
\hline \multicolumn{4}{|c|}{ SOC in $\mathrm{WSA}_{\mathrm{ma}} 3-2 \mathrm{~mm}$} \\
\hline $\mathrm{G}$ & $y=-0.275 x+568.1$ & n.s. & decrease \\
\hline $\mathrm{T}$ & $y=0.9298 x-1855.3$ & n.s. & increase \\
\hline $\mathrm{T}+\mathrm{FYM}$ & $y=0.6762 x-1342.4$ & + & increase \\
\hline $\mathrm{G}+\mathrm{NPK} 3$ & $y=0.1964 x-377.75$ & n.s. & increase \\
\hline $\mathrm{G}+\mathrm{NPK} 1$ & $y=-0.456 x+933.4$ & n.s. & decrease \\
\hline \multicolumn{4}{|c|}{ SOC in $\mathrm{WSA}_{\mathrm{ma}} 2-1 \mathrm{~mm}$} \\
\hline $\mathrm{G}$ & $y=0.0536 x-91.346$ & n.s. & increase \\
\hline $\mathrm{T}$ & $y=0.9738 x-1943.4$ & + & increase \\
\hline $\mathrm{T}+\mathrm{FYM}$ & $y=0.8905 x-1771.9$ & n.s. & increase \\
\hline $\mathrm{G}+\mathrm{NPK} 3$ & $y=0.0321 x-47.468$ & n.s. & increase \\
\hline $\mathrm{G}+\mathrm{NPK} 1$ & $\mathrm{y}=-0.2536 \mathrm{x}+527.47$ & n.s. & decrease \\
\hline \multicolumn{4}{|c|}{ SOC in $\mathrm{WSA}_{\mathrm{ma}} 1-0.5 \mathrm{~mm}$} \\
\hline G & $y=0.056 x-95.786$ & n.s. & increase \\
\hline $\mathrm{T}$ & $\mathrm{y}=1.2167 \mathrm{x}-2432.8$ & + & increase \\
\hline $\mathrm{T}+\mathrm{FYM}$ & $y=0.9083 x-1808.3$ & n.s. & increase \\
\hline $\mathrm{G}+\mathrm{NPK} 3$ & $y=-0.2238 x+466.49$ & n.s. & decrease \\
\hline $\mathrm{G}+\mathrm{NPK} 1$ & $y=0.9369 x-1867.4$ & n.s. & increase \\
\hline \multicolumn{4}{|c|}{ SOC in WSA ${ }_{\mathrm{ma}} 0.5-0.25 \mathrm{~mm}$} \\
\hline G & $y=-0.5381 x+1096.4$ & n.s. & decrease \\
\hline $\mathrm{T}$ & $y=0.775 x-1546.5$ & + & increase \\
\hline $\mathrm{T}+\mathrm{FYM}$ & $y=0.394 x-776.61$ & n.s. & increase \\
\hline $\mathrm{G}+\mathrm{NPK} 3$ & $y=-0.6095 x+1241$ & n.s. & decrease \\
\hline $\mathrm{G}+\mathrm{NPK} 1$ & $y=0.3405 x+699.87$ & n.s. & decrease \\
\hline \multicolumn{4}{|c|}{ SOC in $\mathrm{WSA}_{\mathrm{mi}}<0.25 \mathrm{~mm}$} \\
\hline G & $y=-0.1488 x+311.52$ & n.s. & decrease \\
\hline $\mathrm{T}$ & $y=0.4155 x-825.69$ & n.s. & increase \\
\hline $\mathrm{T}+\mathrm{FYM}$ & $y=0.1488 x-287.19$ & n.s. & increase \\
\hline $\mathrm{G}+\mathrm{NPK} 3$ & $y=0.3762 x-744.11$ & n.s. & increase \\
\hline $\mathrm{G}+\mathrm{NPK} 1$ & $y=0.0881 x-164.55$ & n.s. & increase \\
\hline
\end{tabular}

where: G - control; FYM - farmyard manure; G + NPK3 - doses of NPK fertilisers in $3^{\text {rd }}$ intensity for vineyards; G +

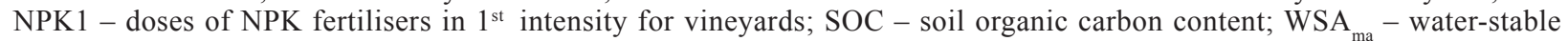
macroaggregates, $\mathrm{WSA}_{\mathrm{mi}}$ - water-stable micro aggregates. ${ }^{++} P \leq 0.01 ;{ }^{+} P \leq 0.05$; n.s. - non-significant. 
T

Trends in the $\mathrm{C}_{\mathrm{NL}}$ distribution in the WSA during the 2008-2015 (y= $\mathrm{C}_{\mathrm{NL}}$ content) with time $(\mathrm{x}=$ years $)$

\begin{tabular}{|c|c|c|c|}
\hline Treatments & Equations & Probability & Trend \\
\hline \multicolumn{4}{|c|}{$\mathrm{C}_{\mathrm{NL}}$ in $\mathrm{WSA}_{\mathrm{ma}}>5 \mathrm{~mm}$} \\
\hline $\mathrm{G}$ & $y=44.917 x-75412$ & n.s. & increase \\
\hline $\mathrm{T}$ & $y=1085.5 x-2 E+06$ & + & increase \\
\hline $\mathrm{T}+\mathrm{FYM}$ & $y=1133.5 x-2 E+06$ & n.s. & increase \\
\hline $\mathrm{G}+\mathrm{NPK} 3$ & $y=736.01 x-1 E+06$ & n.s. & increase \\
\hline $\mathrm{G}+\mathrm{NPK} 1$ & $y=489.43 x-969377$ & n.s. & increase \\
\hline \multicolumn{4}{|c|}{$\mathrm{C}_{\mathrm{NL}}$ in WSA $\mathrm{Wa}_{\mathrm{ma}} 5-3 \mathrm{~mm}$} \\
\hline $\mathrm{G}$ & $y=-50.5 x+114625$ & n.s. & decrease \\
\hline $\mathrm{T}$ & $y=981.35 x-2 E+06$ & + & increase \\
\hline $\mathrm{T}+\mathrm{FYM}$ & $y=921.99 x-2 E+06$ & + & increase \\
\hline $\mathrm{G}+\mathrm{NPK} 3$ & $y=225 x-437456$ & n.s. & increase \\
\hline $\mathrm{G}+\mathrm{NPK} 1$ & $y=-500.77 x+1 E+06$ & n.s. & decrease \\
\hline \multicolumn{4}{|c|}{$\mathrm{C}_{\mathrm{NL}}$ in WSA $\mathrm{Wa}_{\mathrm{ma}} 3-2 \mathrm{~mm}$} \\
\hline G & $y=-196.46 x+408392$ & n.s. & decrease \\
\hline $\mathrm{T}$ & $y=751.01 x-1 E+06$ & n.s. & increase \\
\hline $\mathrm{T}+\mathrm{FYM}$ & $y=636.33 x-1 E+06$ & + & increase \\
\hline $\mathrm{G}+\mathrm{NPK} 3$ & $y=174.69 x-336253$ & n.s. & increase \\
\hline $\mathrm{G}+\mathrm{NPK} 1$ & $y=-453.45 x+926357$ & n.s. & decrease \\
\hline \multicolumn{4}{|c|}{$\mathrm{C}_{\mathrm{NL}}$ in $\mathrm{WSA}_{\mathrm{ma}} 2-1 \mathrm{~mm}$} \\
\hline G & $y=77.429 x-141240$ & n.s. & increase \\
\hline $\mathrm{T}$ & $y=862.48 x-2 E+06$ & + & increase \\
\hline $\mathrm{T}+\mathrm{FYM}$ & $y=739.76 x-1 E+06$ & n.s. & increase \\
\hline $\mathrm{G}+\mathrm{NPK} 3$ & $y=16.167 x-17530$ & n.s. & increase \\
\hline $\mathrm{G}+\mathrm{NPK} 1$ & $y=-203.48 x+424716$ & n.s. & decrease \\
\hline \multicolumn{4}{|c|}{$\mathrm{C}_{\mathrm{NL}}$ in $\mathrm{WSA}_{\mathrm{ma}} 1-0.5 \mathrm{~mm}$} \\
\hline G & $y=72.071 x-130292$ & n.s. & increase \\
\hline $\mathrm{T}$ & $y=1199.1 x-2 E+06$ & + & increase \\
\hline $\mathrm{T}+\mathrm{FYM}$ & $y=912.48 x-2 E+06$ & n.s. & increase \\
\hline $\mathrm{G}+\mathrm{NPK} 3$ & $y=-223.36 x+463164$ & n.s. & decrease \\
\hline $\mathrm{G}+\mathrm{NPK} 1$ & $y=874.26 x-2 E+06$ & n.s. & increase \\
\hline \multicolumn{4}{|c|}{$\mathrm{C}_{\mathrm{NL}}$ in $\mathrm{WSA}_{\mathrm{ma}} 0.5-0.25 \mathrm{~mm}$} \\
\hline $\mathrm{G}$ & $y=-424.3 x+865686$ & n.s. & decrease \\
\hline $\mathrm{T}$ & $y=750.8 x-1 E+06$ & + & increase \\
\hline $\mathrm{T}+\mathrm{FYM}$ & $y=301.56 x-592793$ & n.s. & increase \\
\hline $\mathrm{G}+\mathrm{NPK} 3$ & $y=-536.06 x+1 E+06$ & n.s. & decrease \\
\hline $\mathrm{G}+\mathrm{NPK} 1$ & $y=-272.33 x+560788$ & n.s. & decrease \\
\hline \multicolumn{4}{|c|}{$\mathrm{C}_{\mathrm{NL}}$ in $\mathrm{WSA}_{\mathrm{mi}}<0.25 \mathrm{~mm}$} \\
\hline $\mathrm{G}$ & $\mathrm{y}=1.5714 \mathrm{x}+7461$ & n.s. & increase \\
\hline $\mathrm{T}$ & $y=433.73 x-864069$ & n.s. & increase \\
\hline $\mathrm{T}+\mathrm{FYM}$ & $y=193 x-377881$ & n.s. & increase \\
\hline $\mathrm{G}+\mathrm{NPK} 3$ & $y=443.81 x-882121$ & n.s. & increase \\
\hline $\mathrm{G}+\mathrm{NPK} 1$ & $y=159.65 x-310174$ & n.s. & increase \\
\hline
\end{tabular}

where: G - control; FYM - farmyard manure; G + NPK3 - doses of NPK fertilisers in $3^{\text {rd }}$ intensity for vineyards; G + NPK1 - doses of NPK fertilisers in $1^{\text {st }}$ intensity for vineyards; $\mathrm{C}_{\mathrm{NL}}-$ non-labile carbon content; WSA $\mathrm{ma}_{\text {a }}$ - water-stable macroaggregates, $\mathrm{WSA}_{\mathrm{mi}}$ - water-stable micro aggregates. ${ }^{++} P \leq 0.01 ;{ }^{+} P \leq 0.05$; n.s. - non-significant. 
$\mathrm{T}$ a

Trends of $\mathrm{C}_{\mathrm{L}}$ distribution in the WSA during the 2008-2015 ( $\mathrm{y}=\mathrm{C}_{\mathrm{L}}$ content) with time $(\mathrm{x}=$ years $)$

\begin{tabular}{|c|c|c|c|}
\hline Treatments & Equations & Probability & Trend \\
\hline \multicolumn{4}{|c|}{$\mathrm{C}_{\mathrm{L}}$ in $\mathrm{WSA}_{\mathrm{ma}}>5 \mathrm{~mm}$} \\
\hline G & $y=-37.774 x+77919$ & n.s. & decrease \\
\hline $\mathrm{T}$ & $y=230.01 x-460149$ & n.s. & increase \\
\hline $\mathrm{T}+\mathrm{FYM}$ & $y=159.33 x-317307$ & n.s. & increase \\
\hline $\mathrm{G}+\mathrm{NPK} 3$ & $y=193.75 x-387052$ & n.s. & increase \\
\hline $\mathrm{G}+\mathrm{NPK} 1$ & $y=15.571 x-29333$ & n.s. & increase \\
\hline \multicolumn{4}{|c|}{$\mathrm{C}_{\mathrm{L}}$ in $\mathrm{WSA}_{\mathrm{ma}} 5-3 \mathrm{~mm}$} \\
\hline G & $y=-63.744 x+130027$ & n.s. & decrease \\
\hline $\mathrm{T}$ & $y=193.65 x-387369$ & ++ & increase \\
\hline $\mathrm{T}+\mathrm{FYM}$ & $y=55.393 x-108862$ & n.s. & increase \\
\hline $\mathrm{G}+\mathrm{NPK} 3$ & $y=39.327 x-76564$ & n.s. & increase \\
\hline $\mathrm{G}+\mathrm{NPK} 1$ & $y=-90.893 x+184796$ & n.s. & decrease \\
\hline \multicolumn{4}{|c|}{$\mathrm{C}_{\mathrm{L}}$ in $\mathrm{WSA}_{\mathrm{ma}} 3-2 \mathrm{~mm}$} \\
\hline G & $y=-78.494 x+159624$ & n.s. & decrease \\
\hline $\mathrm{T}$ & $y=177.32 x-354572$ & n.s. & increase \\
\hline $\mathrm{T}+\mathrm{FYM}$ & $y=39.905 x-77946$ & n.s. & increase \\
\hline $\mathrm{G}+\mathrm{NPK} 3$ & $y=21.78 x-41584$ & n.s. & increase \\
\hline $\mathrm{G}+\mathrm{NPK} 1$ & $y=-2.5 x+7054$ & n.s. & decrease \\
\hline \multicolumn{4}{|c|}{$\mathrm{C}_{\mathrm{L}}$ in $\mathrm{WSA}_{\mathrm{ma}} 2-1 \mathrm{~mm}$} \\
\hline G & $y=-23.815 x+49810$ & n.s. & decrease \\
\hline $\mathrm{T}$ & $y=111.33 x-221516$ & + & increase \\
\hline $\mathrm{T}+\mathrm{FYM}$ & $y=150.71 x-300501$ & n.s. & increase \\
\hline $\mathrm{G}+\mathrm{NPK} 3$ & $y=15.976 x-29938$ & n.s. & increase \\
\hline $\mathrm{G}+\mathrm{NPK} 1$ & $y=-50.054 x+102672$ & n.s. & decrease \\
\hline \multicolumn{4}{|c|}{$\mathrm{C}_{\mathrm{L}}$ in $\mathrm{WSA}_{\mathrm{ma}} 1-0.5 \mathrm{~mm}$} \\
\hline G & $y=-16.077 x+34423$ & n.s. & decrease \\
\hline $\mathrm{T}$ & $y=-7.9762 x+17974$ & n.s. & decrease \\
\hline $\mathrm{T}+\mathrm{FYM}$ & $y=-4.1429 x+10769$ & n.s. & decrease \\
\hline $\mathrm{G}+\mathrm{NPK} 3$ & $y=-0.4107 x+3245,1$ & n.s. & decrease \\
\hline $\mathrm{G}+\mathrm{NPK} 1$ & $y=62.66 x-123608$ & n.s. & increase \\
\hline \multicolumn{4}{|c|}{$\mathrm{C}_{\mathrm{L}}$ in $\mathrm{WSA}_{\mathrm{ma}} 0.5-0.25 \mathrm{~mm}$} \\
\hline G & $y=-113.76 x+230584$ & n.s. & decrease \\
\hline $\mathrm{T}$ & $y=24.202 x-46758$ & n.s. & increase \\
\hline $\mathrm{T}+\mathrm{FYM}$ & $y=111.54 x-221936$ & n.s. & increase \\
\hline $\mathrm{G}+\mathrm{NPK} 3$ & $y=-73.423 x+149775$ & n.s. & decrease \\
\hline $\mathrm{G}+\mathrm{NPK} 1$ & $y=-68.101 x+138996$ & n.s. & decrease \\
\hline \multicolumn{4}{|c|}{$\mathrm{C}_{\mathrm{L}}$ in $\mathrm{WSA}_{\mathrm{mi}}$} \\
\hline G & $y=-150.38 x+304056$ & + & decrease \\
\hline $\mathrm{T}$ & $y=-18.25 x+38377$ & n.s. & decrease \\
\hline $\mathrm{T}+\mathrm{FYM}$ & $y=-44.19 x+90688$ & n.s. & decrease \\
\hline $\mathrm{G}+\mathrm{NPK} 3$ & $y=-67.619 x+138014$ & n.s. & decrease \\
\hline $\mathrm{G}+\mathrm{NPK} 1$ & $y=-71.56 x+145621$ & n.s. & decrease \\
\hline
\end{tabular}

where: G - control; FYM - farmyard manure; G + NPK3 - doses of NPK fertilisers in $3^{\text {rd }}$ intensity for vineyards; G + NPK1 - doses of NPK fertilisers in $1^{\text {st }}$ intensity for vineyards; $\mathrm{C}_{\mathrm{L}}$ - labile carbon content; $\mathrm{WSA}_{\mathrm{ma}}$ - water-stable macroaggregates, WSA $_{\mathrm{mi}}-$ water-stable micro aggregates. ${ }^{++} P \leq 0.01 ;{ }^{+} P \leq 0.05 ;$ n.s. - non-significant. 
as compared to unfertilised soil (Shimizu et al. 2009). This indicates $C$ loss which is connected with the higher use of chemical fertilisers - especially nitrogen (Edwards et al. 1992; Yang 2011). On the other hand, higher nutrient contents through biomass production can increase SOC in the higher size fractions of $\mathrm{WSA}_{\mathrm{ma}}$, especially in the shortterm (Šimanský \& Polláková 2012). As mentioned above, overall the highest average content of SOC in $\mathrm{WSA}_{\mathrm{ma}}$ was determined in the T+FYM treatment (Table 2). However, application of FYM (40 t/ha) significantly built-up SOC in $\mathrm{WSA}_{\mathrm{ma}}$ at an average rate of 0.98 and $0.68 \mathrm{~g} / \mathrm{kg} / \mathrm{y}$ for only the size fractions 5-3 $\mathrm{mm}$ and 3-2 $\mathrm{mm}$, respectively (Table 3 ). Expressed as percentage, this is an increase of 44, and $30 \%$ of SOC in these size fractions of WSA over the period of 8 years. Results of our study have shown that the highest carbon sequestration after the FYM application is connected with the higher size fractions of $\mathrm{WSA}_{\mathrm{ma}}$. Similar results were found in the studies of Kundu et al. (2007) and Huang et al. (2010). The study of Tong et al. (2014) found there to be increased amplitude of stock and sequestration rate in $\mathrm{C}$ under FYM, than for mineral fertiliser. Our results confirm this findings (Table 2).

\section{Non-labile carbon in water-stable aggregates}

Soil organic-carbon pools can be divided into a labile pool and a recalcitrant fraction (Belay-Tedla et al. 2009). Both pools were determined in this study. The content of non-labile carbon $\left(\mathrm{C}_{\mathrm{NL}}\right)$ in WSA, which represents a large sized pool and slow response to soil micro-organism activity, is shown in Table 2. The $\mathrm{C}_{\mathrm{NL}}$ contents in WSA ranged from 84 to $89 \%$ (from SOC) and the effect of soil management practices on $\mathrm{C}_{\mathrm{NL}}$ in WSA were significant. The $\mathrm{C}_{\mathrm{NL}}$ contents reflected the contents of SOC in WSA. Overall, the highest average content of $\mathrm{C}_{\mathrm{NL}}$ in WSA $_{\text {ma }}$ was found for the T+FYM treatment, while the lowest ones were in the $\mathrm{T}$ treatment. The content of $\mathrm{C}_{\mathrm{NL}}$ in $\mathrm{WSA}_{\mathrm{ma}}$ increased on average in the following order: $\mathrm{T}<\mathrm{G}<\mathrm{G}+\mathrm{NPK} 1<\mathrm{G}+\mathrm{NPK} 3<\mathrm{T}+\mathrm{FYM}$. The dynamics of $\mathrm{C}_{\mathrm{NL}}$ at individual aggregate sizes of $\mathrm{WSA}_{\mathrm{ma}}$ showed that intensive tillage significantly increased $\mathrm{C}_{\mathrm{NL}}$ in $\mathrm{WSA}_{\mathrm{ma}}$. This was especially so in the last two smallest size fractions of $\mathrm{WSA}_{\mathrm{ma}} 1-0.5$ and $0.5-0.25 \mathrm{~mm}$, except for the size fraction of 3-2 $\mathrm{mm}$ during the whole period (Table 4). The same was found in case of WSA $\mathrm{mi}_{\mathrm{i}}$. Application of FYM significantly built-up the $\mathrm{C}_{\mathrm{NL}}$ in $\mathrm{WSA}_{\mathrm{ma}}$ at an average rate of 0.92 and $0.64 \mathrm{~g} / \mathrm{kg} / \mathrm{y}$ across the size fractions of 5-3 mm and 3-2 $\mathrm{mm}$, respectively. That means increase of $152 \%$ and $132 \%$ for $\mathrm{C}_{\mathrm{NL}}$ in $\mathrm{WSA}_{\mathrm{ma}}$ in 5-3 $\mathrm{mm}$ and 3-2 $\mathrm{mm}$ over the period. The values of $\mathrm{C}_{\mathrm{NL}}$ after the application of mineral fertilisers $(\mathrm{G}+\mathrm{NPK} 1$ a G+NPK3) had great fluctuations during the individual years (2008-2015) and therefore it was not possible to estimate the trend in the change of their values for the individual aggregate size fractions.

\section{Labile carbon in water-stable aggregates}

Tillage had no significant effect on the $\mathrm{C}_{\mathrm{L}}$ content in $\mathrm{WSA}_{\mathrm{mi}}$ and the average content of $\mathrm{WSA}_{\mathrm{ma}}$ (Table 2). However, when evaluating the $C_{L}$ at different aggregate sizes of $\mathrm{WSA}_{\mathrm{ma}}$, there was found to be a significant difference in the $\mathrm{C}_{\mathrm{L}}$ content in $\mathrm{WSA}_{\text {ma }}$ 1-2 mm between $\mathrm{T}, \mathrm{G}$ and $\mathrm{G}+\mathrm{NPK} 1$ treatments. In the $\mathrm{T}$ treatment, the average content of $\mathrm{C}_{\mathrm{L}}$ in $\mathrm{WSA}_{\mathrm{mi}}$ and $\mathrm{C}_{\mathrm{L}}$ in $\mathrm{WSA}_{\text {ma }}$ was $1.67 \mathrm{~g} / \mathrm{kg}$ and $2.08 \mathrm{~g} / \mathrm{kg}$, respectively (Table 2). The trend line in Table 5 shows that the $C_{L}$ increase rate in the cultivated treatment ranged from $24 \mathrm{mg} / \mathrm{kg} / \mathrm{y}$ (WSA ${ }_{\text {ma }} 0.5-0.25 \mathrm{~mm}$ ) to $230 \mathrm{mg} / \mathrm{kg} / \mathrm{y}\left(\mathrm{WSA}_{\mathrm{ma}}>5 \mathrm{~mm}\right)$. However there was found to be a significant increase of $\mathrm{C}_{\mathrm{L}}$ content only in the size fractions of $5-3 \mathrm{~mm}$ and $2-1 \mathrm{~mm}$ over the period.

The T+FYM treatment was found to have the highest $\mathrm{C}_{\mathrm{L}}$ in all individual size fractions of $\mathrm{WSA}_{\mathrm{ma}}$ (Table 2). The results are consistent with the findings of Purakayastha et al. (2008) and Abdollahi et al. (2014), where the application of FYM increased the labile C content. Application of FYM decreased $\mathrm{C}_{\mathrm{L}}$ in WSA at an average rate of -4 and $-44 \mathrm{mg} / \mathrm{kg} / \mathrm{y}$ in the size fractions of $1-0.5 \mathrm{~mm}$ and $<0.25 \mathrm{~mm}$, respectively. However there was no statistical significance (Table 5). The built up of $C_{L}$ in the WSA showed great fluctuations, which was caused by the timing of the FYM application. Overall, the highest $\mathrm{C}_{\mathrm{L}}$ content was, however found in the size fraction of $\mathrm{WSA}_{\mathrm{ma}}$ $2-1 \mathrm{~mm}$, which is in line with the study of Abdollahi et al. (2014). Also Degens (1997) found higher $\mathrm{C}_{\mathrm{L}}$ contents in the aggregate size of $>1 \mathrm{~mm}$. Zhang \& Peng (2006) reported an increase of $C_{L}$ in WSA $>5 \mathrm{~mm}$ due to FYM application. This is an aggregation effect which creates the space for sequestration of higher $\mathrm{C}_{\mathrm{L}}$ contents as found in our study (Table 5). 
There were significant differences between the $\mathrm{G}$ and $\mathrm{G}+\mathrm{NPK} 3$ treatments in the $\mathrm{C}_{\mathrm{L}}$ in $\mathrm{WSA}_{\mathrm{mi}}$ and average content of $\mathrm{C}_{\mathrm{L}}$ in $\mathrm{WSA}_{\mathrm{ma}}$. Generally, the application of higher NPK doses resulted in higher contents of $\mathrm{C}_{\mathrm{L}}$ in $\mathrm{WSA}_{\mathrm{ma}}$ and in $\mathrm{WSA}_{\mathrm{mi}}$, as compared to the lower doses of mineral fertiliser (Table 2). The dynamics of the $\mathrm{C}_{\mathrm{L}}$ in WSA were influenced by the different NPK doses in the vineyard during the 2008-2015 (Table 5). Application of NPK at the $3^{\text {rd }}$ intensity of fertilisation builds up $\mathrm{C}_{\mathrm{L}}$ in WSA at an average rate of $193,39,21$ and $15 \mathrm{mg} / \mathrm{kg} / \mathrm{y}$ across the size fractions of $>5 \mathrm{~mm}, 5-3 \mathrm{~mm}, 3-2 \mathrm{~mm}$ and 2-1 mm, respectively. Again however, there was no statistical significance. With the NPK treatment we observed a decline of $\mathrm{C}_{\mathrm{L}}$ in WSA at an average rate of $-0.4,-73$ and $-67 \mathrm{mg} / \mathrm{kg} / \mathrm{y}$ across the size fractions of $1-0.5 \mathrm{~mm}, 0.5-0.25 \mathrm{~mm}$ and $<0.25 \mathrm{~mm}$, respectively, but again with no statistical significance. Our data also showed that in G+NPK1 the most intensive increase of $\mathrm{C}_{\mathrm{L}}$ in $\mathrm{WSA}_{\mathrm{ma}}$ in the size fraction of $1-0.5 \mathrm{~mm}(63 \mathrm{mg} / \mathrm{kg} / \mathrm{y})$. The greatest decline was in the size fraction of $5-3 \mathrm{~mm}(-91 \mathrm{mg} / \mathrm{kg} / \mathrm{y})$ (Table 5). Conteh et al. (1999) reported that $\mathrm{C}_{\mathrm{L}}$ in the soil is related to the contents of fulvic acids, soil polysaccharides and soil microbial biomass carbon. Variable rhizodeposition, a major source of labile carbon in the soil during the year, as well as microbial activity, cause the fluctuation of $\mathrm{C}_{\mathrm{L}}$ production in the soil. This will be influenced by fertilisation (Šimanský 2013). Hence, microbial communities of decomposers in the soil can prefer labile fractions of organic matter as a carbon source. This is positively reflected in aggregation (Shepherd et al. 2001) and might result in a better physical protection also of the labile $\mathrm{C}$ inside the aggregates (Peth et al. 2008).

\section{CONCLUSION}

Our study emphasizes the importance of soil-management practices in relation to carbon sequestration, mainly in water-stable aggregates, for a commercial vineyard. Water-stable aggregates are able to protect carbon. So in this regard it is necessary to pay further attention to their stability, especially in relation of intensive cultivation of vineyards. Water-stable micro-aggregates might be responsible for carbon sequestration in the inten- sive tilled and fertilised vineyard soils. The results of our study indicate that the application of farmyard manure increases the contents of soil organic carbon and both its labile and non-labile forms in water-stable aggregates. Significant differences in the dynamics of carbon in water-stable aggregates indicate the merit of their use as a sensitive indicator of the quality of the soil environment under the different soil management practices. This information is very important for winegrowers. Because on this basis, they can optimize soil-management practices, and avoid environmental degradation of their soils. Based on our findings, we recommend a decrease in the application of high doses of NPK in $3^{\text {rd }}$ intensity of fertilisation for vineyards which means low than $125 \mathrm{~kg} / \mathrm{ha} \mathrm{N}, 50 \mathrm{~kg} / \mathrm{ha} \mathrm{P}$ and $185 \mathrm{k} / \mathrm{ha} \mathrm{K}$, as well as less intensive vineyard soils cultivation. Application of farmyard manure at rate of $40 \mathrm{t} / \mathrm{ha}$ in 3-4 yearly cycles and lower amounts of mineral fertilisers than $100 \mathrm{~kg} / \mathrm{ha} \mathrm{N}, 30 \mathrm{~kg} / \mathrm{ha} \mathrm{P}$ and $120 \mathrm{k} / \mathrm{ha} \mathrm{K}\left(1^{\text {st }}\right.$ intensity of fertilisation for vineyards) would be more suitable for sustainable management with respect to carbon sequestration.

Acknowledgements. The project was supported by the Cultural and Educational Grant Agency of the Ministry of Education, Science, Research and Sport of the Slovak Republic (No. 014SPU-4/2016).

\section{REFERENCES}

ABDOLLAHI, L. - SCHJØNNING, P. - ELMHOLT, S. MUNKHOLM, L.J. 2014. The effects of organic matter application and intensive tillage and traffic on soil structure formation and stability. In Soil \& Tillage Research, vol. 136, pp. 28-37. DOI: 10.1016/S0167-1987(97)00038-X

BELAY-TEDLA, A. - ZHOU, X. - SU, B. - WAN, S. - LUO, Y. 2009. Labile, recalcitrant, and microbial carbon and nitrogen pools of a tallgrass prairie soil in the US Great Plains subjected to experimental warming and clipping. In Soil Biology \& Biochemistry, vol. 41 no. 1, pp. 110-116. DOI: $10.1016 /$ j.soilbio.2008.10.003

BERHE, A.A. - KLEBER M. 2013. Erosion, deposition, and the persistence of soil organic matter: mechanistic considerations and problems with terminology. In Earth Surface Processes and Landforms, vol. 38, no. 8, pp. 908912. DOI: $10.1002 /$ esp.3408

BHATTACHARYYA, R. - VED PRAKASH KUNDU, S. SRIVASTVA, A.K. - GUPTA, H.S. 2010. Long term effects of fertilisation on carbon and nitrogen sequestration and aggregate associated carbon and nitrogen in the Indian subHimalayas. In Nutrient Cycling in Agroecosystems, vol. 86, no. 1, pp. 1-16. DOI: 10.1007/s10705-009-9270-y 
BISWAS, A.K. - MOHANTY, M. - HATI, K.M. - MISRA, A.K. 2009. Distillery effluents effect on soil organic carbon and aggregate stability of a Vertisol in India. In Soil \& Tillage Resarch, vol. 104, no. 2, pp. 241-246. DOI: 10.1016/j.still.2009.02.012

CHAPLOT, V. - COOPER, M. 2015. Soil aggregate stability to predict organic carbon outputs from soils. In Geoderma, vol. 243-244, pp. 205-213. DOI: 10.1016/j. geoderma.2014.12.013

CONTEH, A. - BLAIR, G.J. - LEFROY, R.D.B. WHITBREAD, A. 1999. Labile organic carbon determined by permangante oxidation and its relationships to other measurements of soil organic carbon. In Humic Substances in the Environment, vol. 1, pp. 3-15.

CZACHOR, H. - CHARYTANOWICZ, M. - GONET, S. - NIEWCZAS, J. - JOZEFACIUK, G. - LICHNER, L. 2015. Impact of long-term mineral and organic fertilizer application on the water stability, wettability and porosity of aggregates obtained from two loamy soils. In European Journal of Soil Science, vol. 66, no. 3, pp. 577-588. DOI: 10.1111/ejss. 12242

DEGENS, B.P. 1997. Macro-aggregation of soils by biological bonding and binding mechanisms and the factors affecting these: a review. In Soil Research, vol. 35, no. 3, pp. 431460. DOI: $10.1071 / \mathrm{S} 96016$

DZIADOWIEC, H. - GONET, S.S. 1999. Przewodnik metodyczny do badań materii organicznej gleb [Methodical guide-book for soil organic matter studies]. Prace Komisji Naukowych Polskiego Towarzystwa Gleboznawczego, N. 120, Komisja chemii gleb, Zespół Materii Organicznej Gleb, N II/16, 65 p.

EDWARDS, J.H. - WOOD, C.W. - THURLOW, D.L. RUF, M.E. 1992. Tillage and crop rotation effects on fertility status of a hapludult soil. In Soil Science Society of American Journal, vol. 56, no. 5, pp. 1577-1582. DOI: 10.2136/sssaj1992.03615995005600050040x

FECENKO, J. - LOŽEK, O. 2000. Výživa a hnojenie polných plodin [Nutrition and fertilization of field crops]. Nitra : SPU, pp. 452.

GAIDA, A.M. - PRZEWLOKA, B. - GAWRYJOLEK, K. 2013. Changes in soil quality associated with tillage system applied. In International Agrophysics, vol. 27, no. 2, pp. 133-141. DOI: 10.2478/v10247-012-0078-7

HAYNES, R.J. - NAIDU, R. 1998. Influence of lime, fertilizer and manure applications on soil organic matter content and soil physical conditions: a review. In Nutrient Cycling in Agroecosystems, vol. 51, no. 2, pp. 123-137. DOI: 10.1023/A:1009738307837

HUANG, S. - PENG, X. - HUANG, Q. - ZHANG, W. 2010. Soil aggregation and organic carbon fractions affected by long-term fertilization in a red soil of subtropical China. In Geoderma, vol. 154, no. 3-4, pp. 364-369. DOI: 10.1016/j. geoderma.2009.11.009

IUSS Working Group WRB. 2014. World reference base for soil resources 2014. International soil classification system for naming soils and creating legends for soil maps. Update 2015. World Soil Resources Reports No. 106, Rome : FAO, pp. 192.

JONCZAK, J. 2014. Effect of land use on the carbon and nitrogen forms in humic horizons of Stagnic Luvisols. In Journal of Elementology, vol. 19, no. 4, pp. 1037-1048. DOI: $10.5601 /$ jelem.2014.19.3.345

KHORRAMDEL, S. - KOOCHEKI, A. - MAHALLATI, M.N. - KHORASANI, R. - GHORBANI, R. 2013. Evaluation of carbon sequestration potential in corn fields with different management systems. In Soil \& Tillage Research, vol. 133, pp. 25-31. DOI: 10.1016/S0167-1987(97)00038-X
KUNDU, S. - BHATTACHARYYA, R. - VED-PRAKASH GHOSH, B.N. - GUPTA, H.S. 2007. Carbon sequestration and relationship between carbon addition and storage under rainfed soybean-wheat rotation in a sandy loam soil of the Indian Himalayas. In Soil \& Tillage Research, vol. 92, no. 1-2, pp. 87-95. DOI: 10.1016/j.still.2006.01.009

ŁOGINOW, W. - WISNIEWSKI, W. - GONET, S.S. CIESCINSKA, B. 1987. Fractionation of organic carbon based on susceptibility to oxidation. In Polish Journal of Soil Science, vol. 20, pp. 47-52.

PAUSTIAN, K. - ANDRÉN, O. - JANZEN, H.H. - LAL, R. - SMITH, P. - TIAN, G. - TIESSEN, H. - VAN NOORDWIJK, M. - WOOMER, P.L. 1997. Agricultural soils as a sink to mitigate $\mathrm{CO}_{2}$ emissions. In Soil Use Management, vol. 13, no. 4, pp. 230-244. DOI: 10.1111/ j.1475-2743.1997.tb00594.x

PETH, S. - HORN, R. - BECKMANN, F. - DONATH, T. FISCHER, J.-SMUCKER,A.J.M. 2008. Three-dimensional quantification of intra-aggregate pore-space features using synchrotron-radiation-based microtomography. In Soil Science Society of American Journal, vol. 72, pp. 897-907. DOI: $10.2136 /$ sssaj2007.0130

PLANTE, A.F. - MCGILL, W.B. 2002. Soil aggregate dynamics and the retention of organic matter in laboratory-incubated soil with differing simulated tillage frequencies. In Soil \& Tillage Research, vol. 66, no. 1, pp. 79-92. DOI: 10.1016/ S0167-1987(02)00015-6

POLIDORI, A. - TURPIN, B.J. - DAVIDSON, C.I. RODENBURG, L.A. - MAIMONE, F. 2008. Organic PM2.5: fractionation by polarity, FTIR spectroscopy, and $\mathrm{OM} / \mathrm{OC}$ ratio for the Pittsburgh aerosol. In Aerosol Science and Techology, vol. 42, no. 3, pp. 233-246. DOI: 10.1080/02786820801958767

PURAKAYASTHA, T.J. - RUDRAPPA, L. - SINGH, D. SWARUP, A. - BHADRARAY, S. 2008. Long-term impact of fertilisers on soil organic carbon pools and sequestration rates in maize-wheat-cowpea cropping system. In Geoderma, vol. 144, no. 1-2, pp. 370-378. DOI: 10.1016/j. geoderma.2007.12.006

RABBI, S.M.F. - WILSON, B.R. - LOCKWOOD, P.V. DANIEL, H. - YOUNG I.M. 2015. Aggregate hierarchy and carbon mineralization in two Oxisols of New South Wales, Australia. In Soil \& Tillage Research, vol. 146, pp. 193-203. DOI: 10.1016/j.still.2014.10.008

SHEPHERD, T.G. - SAGGAR, S. - NEWMAN, R.H. - ROSS, C.W. - DANDO, J.L. 2001. Tillage-induced changes to soil structure and organic carbon fraction in New Zealand soils. In Australian Journal of Soil Research, vol. 39, pp. 465-489.

SHIMIZU, M.M. - MARUTANI, S. - DESYATKIN, A.R. - JIN, T.J. - HATA, H. - HATANO, R. 2009. The effect of manure application on carbon dynamics and budgets in a managed grassland of Southern Hokkaido, Japan. In Agriculture, Ecosysems and Environment, vol. 130, no. 1-2, pp. 31-40. DOI: 10.1016/j.agee.2008.11.013

ŠIMANSKÝ, V. 2013. Soil organic matter in water-stable aggregates under different soil management practices in a productive vineyard. Archives of Agronomy and Soil Science, vol. 59, no. 9, pp. 1207-1214. DOI: 10.1080/03650340.2012.708103

ŠIMANSKÝ, V. - BAJČAN, D. 2014. The stability of soil aggregates and their ability of carbon sequestration. In Soil \& Water Research, vol. 9, no. 3, pp. 111-118.

ŠIMANSKÝ, V. - POLLÁKOVÁ, N. 2012. Use of "progressive" soil organic matter parameters for studying of its changes in aggregates in a vineyard. In Acta fytotechnica et zootechnica, vol. 15, no. 4, pp. 109-112. 
ŠIMANSKÝ, V. - TOBIAŠOVÁ, E. - CHLPÍK, J. 2008. Soil tillage and fertilization of Orthic Luvisol and their influence on chemical properties, soil structure stability and carbon distribution in water-stable macro-aggregates. In Soil \& Tillage Research, vol. 100, no. 1-2, pp. 125-132. DOI: 10.1016/j.still.2008.05.008

SIX, J. - BOSSUYT, H. - DEGRYZE, S. - DENEF, K. 2004. A history of research on the link between (micro)aggregates, soil biota, and soil organic matter dynamics. In Soil and Tillage Research, vol. 79, pp. 7-31. DOI: 10.1016/j. still.2004.03.008

TONG, X. - XU, M. - WANG, X. - BHATTACHARYYA, R. - ZHANG, W. - CONG, R. 2014. Long-term fertilisation effects on organic carbon fractions in a red soil of China. In Catena, vol. 113, pp. 251-259. DOI: 10.1016/j. catena.2013.08.005

TRIBERTI, L. - NASTRI, A. - GIORDANI, G. - COMELLINI, F. - BALDONI, G. - TODERI, G. 2008. Can mineral and organic fertilisation help sequestrate carbon dioxide in cropland? In European Journal of Agronomy, vol. 29, no. 1, pp. 13-20. DOI: 10.1016/j.eja.2008.01.009
VADJUNINA, A.F. - KORCHAGINA, Z.A. 1986. Methods of study of soil physical properties. Moscow : Agropromizdat, $415 \mathrm{p}$.

WANG, Y. - ZHANG, J.H. - ZHANG, Z.H. 2015. Influences of intensive tillage on water-stable aggregate distribution on a steep hillslope. In Soil \& Tillage Research, vol. 151, pp. 82-92. DOI: 10.1016/j.still.2015.03.003

WHALEN, J.K. - CHANG, C. 2002. Macroaggregate characteristics in cultivated soils after 25 annual manure applications. In Soil Science Society of American Journal, vol. 66, no. 5, pp. 1637-1647. DOI: 10.2136/sssaj2002.1637

YANG, X.Y. - LI, P.R. - ZHANG, S.L. - SUN, B.H. - CHEN, X.P. 2011. Long-term-fertilisation effects on soil organic carbon, physical properties, and wheat yield of a loess soil. In Journal of Plant Nutrition and Soil Science, 174, pp. 775-784. DOI: 10.1002/jpln.201000134.

ZHANG, B. - PENG, X.H. 2006. Organic matter enrichment and aggregate stabilization in a severely degraded Ultisol after reforestation. In Pedosphere, vol. 16, no. 6, pp. 699706. DOI: $10.1016 / \mathrm{S} 1002-0160(06) 60105-7$

Received: August 11, 2017 